\title{
Direction of Dependence Between Specific Symptoms of Depression : A Non-Gaussian Approach
}

\section{García Velázquez, Regina}

2020-03

García Velázquez , R, Jokela , M \& Rosenstrom , T H 2020 , ' Direction of Dependence Between Specific Symptoms of Depression : A Non-Gaussian Approach ' , Clinical psychological science , vol. 8 , no. 2 , 2167702619875410 , pp. 240-251 . https://doi.org/10.1177/216770261987541

http://hdl.handle.net/10138/325154

https://doi.org/10.1177/2167702619875410

submittedVersion

Downloaded from Helda, University of Helsinki institutional repository.

This is an electronic reprint of the original article.

This reprint may differ from the original in pagination and typographic detail.

Please cite the original version. 


\section{Clinical \\ Psychological SCIENCE

\section{Direction of dependence between specific symptoms of depression: a non-Gaussian approach.}

\begin{tabular}{|c|c|}
\hline Journal: & Clinical Psychological Science \\
\hline Manuscript ID & CPX-18-0319.R1 \\
\hline Manuscript Type: & Empirical Article \\
\hline $\begin{array}{r}\text { Date Submitted by the } \\
\text { Author: }\end{array}$ & $n / a$ \\
\hline Complete List of Authors: & $\begin{array}{l}\text { García-Velázquez, Regina; University of Helsinki, Psychology and } \\
\text { Logopedics } \\
\text { Jokela, Markus; University of Helsinki, Psychology and Logopedics } \\
\text { Rosenström, Tom; University of Helsinki, Psychology and Logopedics }\end{array}$ \\
\hline Keywords: & $\begin{array}{l}\text { direction of dependence, Depression, symptoms, DirectLiNGAM, } \\
\text { Epidemiology }\end{array}$ \\
\hline Abstract: & $\begin{array}{l}\text { Psychopathology could arise from direct interactions between symptoms. } \\
\text { Evidence suggests that the mechanisms underlying somatic and } \\
\text { cognitive-affective symptoms of depression are different. The aim of this } \\
\text { study was to explore dynamic associations among cognitive-affective } \\
\text { depression criteria. For this, we used distribution-based direction of } \\
\text { dependence models, which estimate whether the presence of symptom A } \\
\text { is more likely to depend on the presence of symptom B than vice versa. } \\
\text { We analyzed six large samples of adults from the United States ( } N=34 \\
963 \text { ) and performed a simulation study to test the performance of the } \\
\text { algorithm with ordinal variables. Our results were consistent with the } \\
\text { literature; depressed mood and anhedonia were reactive to changes in } \\
\text { other symptoms, whereas suicidality may reinforce others or reflect } \\
\text { factors doing so. We discuss the results in the context of other literature, } \\
\text { reflect on the potential of these methods in psychopathology, and } \\
\text { consider some practical implications. }\end{array}$ \\
\hline
\end{tabular}

\section{SCHOLARONE Manuscripts}




\section{Direction of dependence between specific symptoms of depression: a non-Gaussian approach}

García-Velázquez, Regina*; Jokela, Markus; Rosenström, Tom Henrik

*Corresponding author:

Department of Psychology and Logopedics, Faculty of Medicine,

P.O. Box 9 (Haartmaninkatu 3), 00014 University of Helsinki, Finland

E-mail: regina.garciavelazquez@helsinki.fi

Word count: $7180+4771=11951$ (this document plus online supplement)

Keywords: direction of dependence; depression; symptoms; DirectLiNGAM; epidemiology 


\begin{abstract}
Psychopathology could arise from direct interactions between symptoms. Evidence suggests that the mechanisms underlying somatic and cognitive-affective symptoms of depression are different. The aim of this study was to explore dynamic associations among cognitive-affective depression criteria. For this, we used distribution-based direction of dependence models, which estimate whether the presence of symptom A is more likely to depend on the presence of symptom B than vice versa. We analyzed six large samples of adults from the United States $(N=34963)$ and performed a simulation study to test the performance of the algorithm with ordinal variables. Our results were consistent with the literature; depressed mood and anhedonia were reactive to changes in other symptoms, whereas suicidality may reinforce other symptoms or reflect factors doing so. We discuss the results in the context of other empirical findings and theories of depression, reflect on the potential of these methods in psychopathology, and consider some practical implications.
\end{abstract}


A growing body of research has focused on how psychopathology could arise from interactions between specific symptoms, rather than being the reflection of a single latent factor causing the symptoms. One of the motivations has been to explain why some disorders co-occur frequently and what gives rise to the observed heterogeneous symptomatology in many disorders. These two issues are particularly important for Major Depression; the disorder varies considerably between individuals in terms of symptom occurrence patterns, prognosis, and response to treatment, and it shows substantial comorbidity with other psychiatric disorders (Fried, 2015; Fried \& Nesse, 2015a, 2015b). The findings concerning depression seem mixed enough to suggest that depression can be under- and overdiagnosed and under- and over-treated at the same time (Lorenzo-Luaces, 2015). This situation motivates research at the symptom level, which is useful in offering more fine-tuned information on the underlying dynamics of psychopathology.

The complex presentation of depression is to some extent understandable because depressive symptoms are diverse, occurring in various domains of human experience (i.e. somatic, affectivemotivational, and cognitive). Depression presents with many different symptoms (Fried, 2017; Kendler, 2016), but its diagnosis is reduced to nine diagnostic criteria in Major Depressive Disorder (MDD), as defined by DSM-5 (APA, 2013). These criteria can be grouped into somatic (weight or appetite changes, disturbed sleep, fatigue, diminished ability to think or concentrate, and psychomotor alterations) and cognitive-affective (depressed mood, anhedonia or loss of interest, feelings of worthlessness or guilt, and recurrent thoughts of death or suicidal ideation) categories.

The somatic symptoms of depression seem to overlap with those of other physical illness. There is ample evidence of these associations at both the dimensional (i.e. symptom sum or 'syndrome') and symptom levels. Somatic, but not cognitive-affective symptoms, independently relate to physical 
conditions like diabetes, metabolic syndrome, cardiac disease, or inflammation (de Jonge, Mangano, \& Whooley, 2007; Hawkins, Callahan, Stump, \& Stewart, 2014; Hwang, Moser, Pelter, Nesbitt, \& Dracup, 2015; Jokela, Virtanen, Batty, \& Kivimäki, 2016; Lamers, Milaneschi, de Jonge, Giltay, \& Penninx, 2018; Messerotti Benvenuti, Buodo, Mennella, \& Palomba, 2015; Michal et al., 2013; Roest et al., 2013; Wiltink et al., 2014, 2018). Thus, it seems difficult to draw the boundary between somatic presentations of depression and other bodily symptoms. Additionally, the somatic criteria of MDD are also present in other mental disorders (e.g. anxiety spectrum), while the cognitive-affective criteria are more specific to MDD. The cognitive/affective symptoms seem to have other correlates. For instance, Jokela et al. (under review) found that social risk factors were associated with almost all of the cognitive/affective symptoms, but only sporadically with somatic symptoms. These patterns point to the possibility that the mechanisms underlying somatic and cognitive-affective clusters are different. The aim of this study is to examine dynamic associations among the cognitive-affective symptoms of depression, which are characteristic of depression and consistently associated with similar risk factors.

Traditional perspectives to psychopathology tend to model syndromes as latent liabilities that cause proneness to suffer from multiple symptoms. In other words, symptoms are considered as indicators of an underlying disease state. This view has been criticized for implying that symptoms have no clinically relevant causal relations, thus giving them a secondary role in psychiatric syndromes (Fried, 2015). This is in contrast to the network approach, which argues that symptoms directly interact with each other, giving rise to a syndrome (Borsboom, 2017). The network approach emphasizes that understanding symptom dynamics is important for orienting treatment decisions, and for preventing a full-blown episode from developing or adopting a self-sustaining state. The dynamics between beliefs and affect have played a key role also in the older cognitive theories of depression (Beck \& Haigh, 2014). However, most of the models currently used for estimating symptom-to-symptom associations 
are typically restricted to analysis of covariances (i.e. the first and second central moments of probability distributions; Kruis \& Maris, 2016; Marsman et al., 2018).

Here, we will use recently developed methodology to extract fruitful information from symptom variables. Instead of showing how symptoms covary with each other, we will explore whether the presence of a specific depressive symptom is informative of the proneness to suffer from the other symptoms. This is a novel approach that can be implemented with Direction of Dependence methods, which aim to detect dominance associations between two or more observed variables using their joint statistical distributions (Dodge \& Rousson, 2000; Shimizu, Hoyer, Hyvärinen, \& Kerminen, 2006; Wiedermann, Kim, Sungur, \& Von Eye, in press; Wiedermann \& Von Eye, 2016). A considerable strength of these methods is the use of non-experimental, cross-sectional data to estimate whether the level of variable A is more likely to depend on the level of variable B than vice versa. This is particularly critical in psychopathology for two reasons. First, the experimental manipulations required to infer dependence often are simply unethical (e.g. inducing symptoms of depression such as suicidal thoughts). Second, other research settings often used for these inferences, such as temporal antecedence studies, are rather costly and not free from controversy (Hamaker, Kuiper, \& Grasman, 2015; Oud \& Delsing, 2010).

Some of the direction of dependence methods are widely applied in specific disciplines, such as brain imaging, but have rarely been applied in the study of psychopathology (Adali, Anderson, \& Fu, 2014; Hyvärinen, Karhunen, \& Oja, 2001; Shimizu, 2018). In this study, we will explore the direction of dependence among the four cognitive-affective diagnostic criteria of MDD. We will use six large cross-sectional samples representative of the adult population in the United States, drawn from the National Health and Nutrition Examination Survey. This will provide six independent replications of an exploratory analysis. We will also perform a simulation study to test the estimation success of the 
algorithm with ordinal variables (e.g. Likert scales), which are typical of questionnaires in psychopathology such as the Patient Health Questionnaire that we used here (Kroenke \& Spitzer, 2002).

\section{Methods}

\section{Participants}

We use the continuous National Health and Nutrition Examination Surveys (NHANES, http://www.cdc.gov/nchs/nhanes/), a collection of comparable cross-sectional samples taken every second year to represent the population in the United States. NHANES studies are described elsewhere (Centers for Disease Control and Prevention, 2017). The samples were from the years 2005, 2007, 2009, 2011, 2013, and $2015(\mathrm{n}=5334,5995,6360,5615,5924$, and 5735, respectively). These samples are all cross-sectional and highly comparable. We conducted the same analyses separately in each of the six samples to determine whether the results replicate across the datasets.

The Patient Health Questionnaire (PHQ-9, Kroenke and Spitzer, 2002) measures depression according to the DSM-5 criteria. It contains one item per criterion, summing up to nine ordinal items, each having four Likert-type options. The items query how often the participant has been bothered by the symptoms in the last two weeks, and the items we used are: "little interest or pleasure in doing things" (corresponding to anhedonia/loss of interest), "feeling down, depressed, or hopeless" (corresponding to depressed mood), "feeling bad about yourself - or that you're a failure or have let yourself or your family down" (corresponding to feelings of worthlessness and guilt), and "thoughts that you would be better off dead or of hurting yourself in some way" (corresponding to thoughts of death and self-harm).

Statistical analysis 
We first chose the Confirmatory Tetrad Test (CTT, Bollen \& Ting, 2000) for testing whether a reflective latent variable model was plausible given the data. If the reflective model was consistent with the data, it would imply that the items (i.e. the symptoms) are independent of each other, conditional on a common latent trait (i.e. depression liability). If this was the case, it would be unwise to conduct symptom-to-symptom analyses because the symptoms appear related to each other only through their associations with a latent common trait. Conversely, a CTT test rejecting the null hypothesis would indicate that the associations between the items are not fully explained by a single common trait, and therefore, it is possible to detect some item-to-item dependencies in a network model. Detailed information about the CTT and the principle of vanishing tetrads can be found in Supplement 1.

For exploring the item-to-item dependencies, we used an algorithm based on the Linear NonGaussian Model (LiNGAM; Shimizu et al., 2006; 2011). Apart from the commonly used covariance structures, there is additional information contained in non-normally distributed data (for an introduction to this method, see Supplement 2). This information can be used to infer which variable most likely has an influence on another (Dodge \& Rousson, 2000; Shimizu, Hoyer, Hyvärinen, \& Kerminen, 2006; Wiedermann, Kim, Sungur, \& Von Eye, in press.; Wiedermann \& Von Eye, 2016). The LiNGAM is particularly well suited for psychopathology research with its requirement of nonGaussianity. Constructs in psychopathology are most accurately modeled by skewed underlying distributions (i.e. most individuals suffer from very few symptoms), which correspond to asymmetric observed variables that hardly ever follow normal distributions (Micceri, 1989; Spirtes \& Zhang, 2016).

The algorithm that we used is DirectLiNGAM. Few epidemiological studies have to date used this algorithm (Helajärvi et al., 2014; Rosenström et al., 2012), but it has shown promising performance in both simulation studies and real-world datasets (Rosenström et al., 2012; Rosenström \& García- 


\begin{abstract}
Velázquez, in press; Shimizu et al., 2011). These studies have addressed the robustness of the algorithm against violations of assumptions such as variable distributions approaching normality, the presence of latent confounding, or considerable measurement error.

\section{Preliminary simulation studies}

We conducted two brief simulation studies. The first one was to inspect how the DirectLiNGAM algorithm performs with ordinal data. This is because the LiNGAM methods have been developed for continuous variables, which have more variability and more accurately defined units than the ordinalvalued (Likert-type) variables. Ordinal items are used very commonly in psychological questionnaires. We have little information on how DirectLiNGAM would perform with ordinal data, except for simulations by Rosenström et al. (2012) showing nearly flawless estimation success under a scalepreserving categorization of continuous variables. A more detailed simulation study is needed to reveal to users the performance of DirectLiNGAM when applied to ordinal variables such as Likert-type scales. The reader can find in Supplement 3 the rationale for conducting the simulation study, the different settings that we tested, and the results. We briefly report here that, according to the simulation results, DirectLiNGAM showed very good estimation success rates for the simulation parameters corresponding to the data used in our empirical example.

The second simulation study, found in Supplement 4, examined the performance of DirectLiNGAM in cases where there was no dependence between the variables analyzed. With this brief simulation we estimated the risk of finding dependence between symptoms that are truly nondependent from each other, which is related to type I error. Note that non-dependent pairs of variables may be or not independent in terms of linear correlation. We compared the distributions of the T statistic for the case of non-dependence and dependence (conditions $\mathrm{X} \perp \mathrm{Y}$ and $\mathrm{X} \rightarrow \mathrm{Y}$ ). The results of this simulation showed that the algorithm correctly detects the absence of distribution-based 
dependence across all data sizes studies, ranging from 100 to 5000 . We thus proceeded with the empirical data analyses.

\section{Empirical data analysis}

The CTT analyses were done based on covariance matrices, instead of polychoric correlation matrices, because the latter assumes the underlying latent variable is normally distributed. This was not the case in our data, for which pairwise and multivariate underlying normality were rejected in statistical tests. To further relax distributional assumptions, the confidence intervals for the non-parametric CTT tests were based on 2000 bootstrap resamples, according to the work by Bollen and Ting (Bollen, 1990; Bollen \& Ting, 1998, 2000). The CTT analyses were conducted using the freely available software R, version 3.4.0. (R Core Team, 2017). For a detailed description of the CTT and the role of tetrads in structural equation models, see Supplement 1.

The DirectLiNGAM algorithm was applied to the four categorical items in each of the NHANES cross-sectional cohorts separately. The algorithm relies on the LiNGAM model, which has three assumptions: (i) the data-generating process is linear and acyclic; (ii) there are no unobserved confounders; and (iii) residuals have non-Gaussian distributions of non-zero variances (Shimizu et al., 2006). Under these assumptions, the dependence relations between variables can be modeled for a single observation (for a study participant) in a matrix form as follows:

$$
x=\mu+B x+\epsilon
$$

where the vector $\mu$ contains the constants and the strength matrix B collects the regression weights. The $\mathrm{B}$ matrix is assumed to be acyclic in the sense that when direction of dependence exists between variables $x_{1}$ and $x_{2}$ it cannot exist between $x_{2}$ and $x_{1}$, and vice versa. Loosely speaking, variables cannot be reciprocally 'causal' for each other, or at least one must directionally dominate the other. The 
vectors $\mathrm{x}$ and $\epsilon$ include the observed variables and residual terms, respectively. The different algorithms relying on the LiNGAM model use different strategies to detect the direction of dependence between two variables and to estimate the weights matrix.

DirectLiNGAM makes use of a nonparametric kernel-based estimate of mutual information (MI; Bach \& Jordan, 2002). Bivariate MI quantifies how much information about one variable can be obtained through the other. It is important to note that MI relates to all dependence, linear and nonlinear, whereas the covariance or Pearson product-moment correlation matrices only entail linear dependence. Also because of the nonparametric kernel estimator, inflation of type I error does not affect the dependence ordering of the variables (i.e. there is no null-hypothesis testing involved). However, it may affect the estimated adjacency matrices, which set the lower triangular coefficients to zero (Thamvitayakul, Shimizu, Ueno, Washio, \& Tashiro, 2012). The reader will find in Supplement 2 an intuitive introduction to the LiNGAM models and to the DirectLiNGAM algorithm. For a technical review on the mathematical grounds of the algorithm, we refer the reader to the original papers by Shimizu and colleagues (Shimizu et al., 2006; 2011).

The analyses were carried out in GNU Octave (Eaton, Bateman, Hauberg, \& Wehbring, 2017), a free software compatible with the DirectLiNGAM 1.2 package developed for Matlab code that is freely provided by the authors (Shimizu, personal website). We used the Dlingamboot function which computes the bootstrap confidence intervals using the percentile method. We set 2000 bootstrap resamples. A sample of the scripts will be made available online (https://bitbucket.org/rgarciav/direction_of_dependence_md/src).

\author{
Results \\ Preliminary simulations
}


The results of the first simulation, which transformed the continuous distributions to ordinal, indicated that DirectLiNGAM was highly accurate in detecting direction of dependence under the conditions of the empirical samples of NHANES studied here. For the results and for a broader view on how DirectLiNGAM functioned with different data, see Supplement 3. Importantly, the algorithm showed problems in some of the settings tested, which caused a noticeable decrease in estimation success. However, we found that DirectLiNGAM yielded a much better estimation success when keeping the smoothing parameters fixed at $\sigma=1.00$ and $\kappa=.02$ for the nonparametric estimation of mutual information. The Discussion section further clarifies the issue and provides recommendations for users.

The results of the second simulation, which examined performance of DirectLiNGAM given pairwise non-dependence, suggest that he risk of finding committing type I error is in practice negligible across the sample sizes studied. See Supplement 4 for more details about the bootstrapped T distributions.

\section{Empirical data analysis}

Descriptive information on the distributions of the items is presented in Table 1. The average symptom intensities were rather low, as expected in general population samples. The CTT analyses based on covariance matrices indicated that the model implications for a reflective model were not met at a $5 \%$ significance level in any of the six cohorts (Table 2). Thus, the unidimensional latent-trait model was invariably rejected in the NHANES datasets, and we proceed to examine direction of dependence across the network of four items.

DirectLiNGAM analyses were consistent across all six datasets. Table 3 displays the total effect estimates of one symptom on another (a total effect includes both direct effect and indirect effect through other symptoms, as elaborated in Supplement 2). The table is read from column to row; the 
directional effect that the symptom in column $j$ has on the symptom in row $i$ is shown in the corresponding cell $i, j$. Empty cells indicate that there is no direct effect of symptom $j$ on symptom $i$. All effects are positive, meaning that a higher rate in a dominant symptom implies a higher score in the estimated consequent symptom. For example, the total effect of "thoughts of death and self-harm" on "anhedonia/loss of interest" in the 2005 dataset is estimated at .79, which includes its direct effect (i.e. thoughts of death and self-harm $\rightarrow$ anhedonia/loss of interest) and the indirect effect through the other symptoms directionally downstream to it (i.e. thoughts of death and self-harm $\rightarrow$ feelings of worthlessness and guilt $\rightarrow$ depressed mood $\rightarrow$ anhedonia/loss of interest). Note, for instance, that the effects over depressed mood are stronger than the effects over anhedonia/loss of interest. Thus, these estimates can be interpreted as regression weights. The dependence sequence derived from Table 3 is illustrated in Figure 1 as a directed acyclic graph.

\section{Discussion}

We studied how the four cognitive-affective criteria of MDD were dependent on each other by using statistical analyses that can derive the direction of dependence between the symptoms from crosssectional data. The algorithm revealed the following directed network: thoughts of death and selfharm $\rightarrow$ feelings of worthlessness and guilt $\rightarrow$ depressed mood $\rightarrow$ anhedonia/loss of interest. The dominance directions were widely consistent across the six samples analyzed, which indicates robustness against sampling process and time (i.e. the datasets were collected every other year between 2005 and 2015). Our findings were in line with several theories of depression and with other empirical findings. We will first review them, and second discuss the role of direction of dependence methods within etiological models. Third, we will describe some clinical implications of the directed network that we found. Fourth, we will list the main limitations of our approach and highlight an important technical aspect of our brief simulation study, and fifth will finish with some conclusions. 
The cognitive model of suicidal behavior, which is embedded within the unified model of depression by Beck and collaborators, posits that once a suicide schema is triggered and activated, there is an increased likelihood that the state of hopelessness continues (Beck \& Bredemeier, 2016; Wenzel \& Beck, 2008). Our results are consistent with the role of the suicide schema in reinforcing negative cognitive processes (i.e. thoughts of death and self-harm $\rightarrow$ feelings of worthlessness and guilt). Also consistent with the direction of dependence found here, in Beck's theory the cognitive processes - including suicide schema and self-criticism - influence affective changes seen in mood and motivation (Beck, 1964). The notion of affective symptoms being reactive to cognition and attributional styles is a common feature of numerous cognitive theories of depression such as the hopelessness theory and Ellis' and Bandura's theories (e.g. Beck \& Haigh, 2014).

Evolutionary theories of depression, such as the bargaining model of depression, state that depression symptoms form a suite of automatic biological responses to situations where the individual has unmet needs or finds herself in a conflict or in an unfair situation (Durisko, Mulsant, \& Andrews, 2015; Hagen, 2003; Hagen \& Rosenström, 2016; Rosenström, 2013). From this point of view, affective and motivational symptoms (i.e. depressed mood and anhedonia/loss of interest) are functional instruments for informing of a disadvantage and reducing the investment on current strategies, while suicidal behavior represents the strongest form of bargaining, understood as an honest signal to others due to high costs to oneself. Preparatory functions associated with affective and motivational symptoms can occur without suicidal thoughts, whereas suicidal thoughts (the extreme response) in depression develop in conjunction with the other symptoms. In other words, suicidal thoughts are a reliable indicator of hardship and of other depressive symptoms, whilst the opposite does not necessarily hold (e.g. low mood in response to romantic loss is perceived as quite normal). Our findings align with this 
theory in that the other residual symptoms can occur independently of suicidality, whereas suicidality more linearly and reliably implies also the other depressive symptoms.

Temporal antecedence is an important criterion for causal inference, although often intricate to interpret when using discrete measurement and when individual trait levels play a role in the temporal processes (e.g. Hamaker, Kuiper, \& Grasman, 2015). One of the reasons is that the estimated lagged effects are specific to the time interval of the study (Kuiper \& Ryan, 2018; Oud \& Delsing, 2010). Although longitudinal studies at the symptom level are scarce, they seem to complement the findings reported in our study. These studies have indicated that suicidality is one of the most stable depressive symptoms over time (meaning it has low variability), while the opposite holds for depressed mood and anhedonia, which vary more across time and react sooner to other symptom changes and to treatment. These studies have taken place over a timespan from a few months to up to nine years (Bringmann, Lemmens, Huibers, Borsboom, \& Tuerlinckx, 2015; Karp et al., 2004; van Eeden, van Hemert, Carlier, Penninx, \& Giltay, in press). Bringmann et al. found in their longitudinal network analysis of depression that, during 14 weekly assessments, suicidal thoughts registered a high level of network outdegree (i.e. were likely to trigger other symptoms) and low indegree (i.e. not likely to be influenced by other symptoms). Additionally, they found that depressed mood and anhedonia are likely to react to changes in other symptoms, but "do not play a large role in funneling the symptom spread themselves" (Bringmann et al., 2015, p. 7).

Thus, suicidality is the most stable symptom in longitudinal studies over long periods of time. However, recent work based on ecological momentary assessment has revealed that death thoughts may fluctuate markedly across short time frames such as over the course of a day (Kleiman et al., 2017). In that study, the authors observed other risk factors for suicidal ideation, such as hopelessness, burdensomeness, and loneliness, to also vary considerably over just a few hours. These symptoms 
correlated with suicidal ideation, but were limited in predicting its short-term changes. In another study, Kleiman et al. (2018) followed participants who had recently attempted suicide. The participants reported their emotions and suicidal symptoms during four weeks via smartphone-based real-time monitoring. The authors found that suicidal thinking leads to downward shifts in mood and concluded that suicidal thoughts may be so persistent for some patients because of their alleviating effect on mood. These studies are novel in providing high-resolution information on suicidality-related symptoms, and illustrate the different pathways for the symptom dynamics suggested in our directed network.

The repeated-measures literature reveals two important aspects. First, intensive longitudinal methods are critical for understanding the temporal dynamics of these cognitive-affective symptoms in more detail. Second, affective symptoms seem to react to suicidality, and not the reverse. This general conclusion was supported by the directed network in this study. In other words, suicidality implies more linearly and reliably the presence of other symptoms than vice versa. In these longitudinal studies, participants diagnosed with depression were followed for a long time or with high temporal resolution. Such studies are very costly to conduct. Here, widely the same symptom dynamics were replicated using different questionnaires, in a community setting, and most importantly using crosssectional samples.

However, it is important to note that direction of dependence does not imply temporal antecedence or other common signs of causality. Instead, dependence here indicates that one direction fits better with a linear model of causation wherein the resulting residual term is statistically (not just linearly) independent of the predictor term, or at least more so than the other direction. When applied to a set of variables, DirectLiNGAM indicates the best-fitting linear acyclic graph. Triangulating the question with different methodology may offer a broader perspective on symptom dynamics. 


\begin{abstract}
"Triangulation" refers to the use of multiple methods with non-overlapping assumptions to guard against errors of inference, which is helpful when developing etiological models in epidemiology (Lawlor, Tilling, \& Smith, 2017; Munafò \& Smith, 2018). Our methodology provides an alternative angle to causation that assists other methodologies by offering testable hypotheses and generating knowledge on alternative signs of causation, often with no extra cost of data collection (i.e. most available data are re-usable for this purpose).
\end{abstract}

A clinical implication of our directed network of symptoms is that preventing and treating biased attributional styles and suicidality may be more effective in producing symptomatic improvements than targeting affective-motivational symptoms like mood, anhedonia, or loss of interest. There is experimental evidence to support the effect of cognitive interventions on emotional symptomatology. Some of these studies have examined techniques used as part of psychotherapy such as cognitive reappraisal or suppression. Reappraisal aims at the reinterpretation of emotion-eliciting events (e.g. images or situational cues) and has been the target of experimental studies for its easy instruction. In brief, individuals are given guidelines to reframe the meaning of stimuli, mostly negative or distressing. In experimental settings, reappraisal is found to reduce self-reported negative emotion, physiological measures of affect, and the functional signal from the amygdala, a brain region involved in affect (see McRae, 2016).

The effects of reappraisal hold also for individuals with remitted and current depression (Kanske, Heissler, Schönfelder, \& Wessa, 2012; Klumpp, Kinney, Bhaumik, \& Fitzgerald, 2018). Cross-cultural studies have found no difference between Western and East Asian natives (Eng, 2012; Tsai, Levenson, \& McCoy, 2006). Moreover, no gender differences exist in the decrease in selfreported negative emotion experience, despite some differential activation of brain areas (Ahn et al., 2015; McRae, Ochsner, Mauss, Gabrieli, \& Gross, 2008). The effects seem to remain after the trial 
(Denny, Inhoff, Zerubavel, Davachi, \& Ochsner, 2015; Denny \& Ochsner, 2014). The downside of the reappraisal technique is that it is more challenging to implement for individuals with clinical depression in day-to-day situations, as compared with the structured laboratory experiments (Visted, Vøllestad, Nielsen, \& Schanche, 2018). Challenges notwithstanding, the ample experimental evidence on cognitive reappraisal inducing changes in emotional response is consistent with other correlational literature highlighting the influence of cognitive factors over emotions, also supported by novel direction of dependence methods.

Our approach has several limitations. Some of the assumptions made by DirectLiNGAM are hardly met in psychopathology research, and consequently, call for caution. The first one is assuming the absence of latent confounders. This implies that the present method cannot distinguish suicidality from its unmeasured liability traits, and thus, the dynamics that we found could reflect some unmeasured latent factor (high neuroticism, borderline personality, etc.). Second, the symptoms were assessed with single items, which entails a higher risk of measurement error compared to the use of several indicators. It has been demonstrated how measurement error can introduce bias, particularly to functional causal model approaches like LiNGAMs (Zhang et al., 2017). In psychopathology, measurement error is highly likely, and it is difficult to quantify its exact influence on our results. However, the method has been found to be robust against measurement errors in previous simulation studies (Hyvärinen, 2010; Rosenström et al., 2012; Rosenström \& García-Velázquez, in press), and here in simulation setting B included in Supplement 3. There are models being developed specifically for accommodating measurement error within the direction of dependence framework (Wiedermann, Merkle, \& Von Eye, 2018).

A third limitation of our approach is acyclicity, a technical assumption of the algorithm which implies an oversimplification in the context of mental health. A thorough simulation study on the 
robustness of DirectLiNGAM to violations of acyclicity would be very useful in the context of psychopathology. Other LiNGAM-based algorithms are being developed, including mixed modeling, which accommodates latent confounding and observation-specific intercepts (Shimizu \& Bollen, 2014). Cyclic relations are inherently difficult to analyze, and thus, it seems wise to start with robust findings on directional dependence and only then build towards more complex models. Acyclicity is a difference between LiNGAM-based graphs and other undirected graphical models containing feedback loops such as the Gaussian Graphical Models. These more parametrized models are often used with high-dimensional, sparsely connected data. In the case of DirectLiNGAM and analyses with a large number of variables, Shimizu et al. $(2011 ; 2012)$ illustrated how to apply adaptive Lasso to prune the directed network. Fourth, our analysis contained a limited number of depression-specific symptoms (i.e. cognitive symptoms of DSM-5). Further research may benefit from including more symptoms, particularly because the exogenous role of suicidal thoughts over the other symptoms may reflect other factors to which suicidality is strongly linked. For example, the distinct link of hopelessness to depressive symptoms and suicidality has been shown by numerous studies (e.g. Baryshnikov et al., 2018; Marchetti, Loeys, Alloy, \& Koster, 2016; Pérez, Marco, \& García-Alandete, 2016; Sokero et al., 2006) and is not measured specifically in PHQ-9.

Our supplementary analysis is the most detailed analysis to date on how accurately DirectLiNGAM functions with ordinal variables. The algorithm showed very high or close to perfect success rates; even with few categories and small sample sizes, the estimation success surpassed $95 \%$. However, we found that the original algorithm published by Shimizu and colleagues does not perform well on ordinal data of sample sizes $\mathrm{N} \geq 1000$ under some circumstances. This can affect detection accuracy and bias results noticeably. We found that, when keeping the smoothing parameters fixed at $\sigma$ $=1.00$ and $\kappa=.02$, DirectLiNGAM registered a much better estimation success. With continuous 
variables, the estimation performance improves as a function of sample size in otherwise similar conditions, but ordinal data require this additional adjustment on behalf of the user. We therefore recommend adjusting the parameters to the above-mentioned values when analyzing ordinal data, and following Shimizu and colleagues' guidelines otherwise (Shimizu et al., 2011, p. 1233).

In conclusion, this study offered an innovative way of exploring symptom-to-symptom dynamics in psychopathology research. The approach generates hypotheses on how depressive symptoms may relate to each other functionally, not only in terms of correlations. Our results were consistent with other empirical evidence and theories of depression; depressed mood and anhedonia are reactive to changes in other symptoms, whereas suicidality may reinforce other symptoms or strongly overlap with factors doing so. These methods have untapped potential in already existing data such as indicating the dominant directions of dependence among psychopathology symptoms, generating testable hypotheses for etiological models, and potentially delineating treatment pathways.

\begin{abstract}
Authorship
R.G-V. and T.R. developed the study concept. All authors planned the contents and structure of the study. R.G-V. performed the data analysis and interpretation under the supervision of T.R. and M.J. The paper was drafted by R.G-V., and T.R. and M.J. provided critical revisions. All authors approved the final version of the paper for submission.
\end{abstract}




\section{References}

Ahn, H. M., Kim, S. A., Hwang, I. J., Jeong, J. W., Kim, H. T., Hamann, S., \& Kim, S. H. (2015). The effect of cognitive reappraisal on long-term emotional experience and emotional memory. Journal of Neuropsychology, 9(1), 64-76. https://doi.org/10.1111/jnp.12035

APA. (2013). Diagnostic and statistical manual of mental disorders (DSM-5®). American Psychiatric Pub.

Bach, F. R., \& Jordan, M. I. (2002). Kernel Independent Component Analysis. Journal of Machine Learning Research, 3, 1-48. Retrieved from https://www.di.ens.fr/ fbach/kernelICA-jmlr.pdf

Baryshnikov, I., Rosenström, T., Jylhä, P., Koivisto, M., Mantere, O., Suominen, K., ... Isometsä, E. T. (2018). State and trait hopelessness in a prospective five-year study of patients with depressive disorders. Journal of Affective Disorders, 239, 107-114. https://doi.org/10.1016/J.JAD.2018.07.007

Beck, A. T., \& Haigh, E. A. P. (2014). Advances in Cognitive Theory and Therapy: The Generic Cognitive Model. Annual Review of Clinical Psychology, 10(1), 1-24. https://doi.org/10.1146/annurev-clinpsy-032813-153734

Bollen, K. (1990). Outlier screening and a distribution-free test for vanishing tetrads. Sociological Methods \& Research, 19(1), 80-92.

Bollen, K. a., \& Ting, K. (1998). Bootstrapping a Test Statistic for Vanishing Tetrads. Sociological Methods \& Research, 27(1), 77-102. https://doi.org/10.1177/0049124198027001002

Bollen, K., \& Ting, K. (2000). A tetrad test for causal indicators. Psychol Methods, 5(1), 3-22. https://doi.org/10.1037/1082-989X.5.1.3 
Borsboom, D. (2017). A network theory of mental disorders. World Psychiatry, 16(1), 5-13.

Bringmann, L. F., Lemmens, L. H. J. M., Huibers, M. J. H., Borsboom, D., \& Tuerlinckx, F. (2015).

Revealing the dynamic network structure of the Beck Depression Inventory-II. Psychological Medicine, 45(04), 747-757. https://doi.org/10.1017/S0033291714001809

Centers for Disease Control and Prevention. (2017). National Health and Nutrition Examination Survey Data (NHANES). Hyattsville, MD: US: Department of Health and Human Services, Centers for Disease Control and Prevention. Retrieved from https://www.cdc.gov/nchs/nhanes/about_nhanes.htm

de Jonge, P., Mangano, D., \& Whooley, M. A. (2007). Differential Association of Cognitive and Somatic Depressive Symptoms With Heart Rate Variability in Patients With Stable Coronary Heart Disease: Findings From the Heart and Soul Study. Psychosomatic Medicine, 69(8), 735739. https://doi.org/10.1097/PSY.0b013e31815743ca

Denny, B. T., Inhoff, M. C., Zerubavel, N., Davachi, L., \& Ochsner, K. N. (2015). Getting Over It: Long-Lasting Effects of Emotion Regulation on Amygdala Response. Psychological Science, 26(9), 1377-1388. https://doi.org/10.1177/0956797615578863

Denny, B. T., \& Ochsner, K. N. (2014). Behavioral effects of longitudinal training in cognitive reappraisal. Emotion, 14(2), 425-433. https://doi.org/10.1037/a0035276

Dodge, Y., \& Rousson, V. (2000). Direction dependence in a regression line. Communications in Statistics - Theory and Methods, 29(9-10), 1957-1972. https://doi.org/10.1080/03610920008832589

Eng, J. S. (2012). Emotion regulation and culture: The effects of cultural models of self on Western and 
East Asian differences in suppression and reappraisal. University of California, Berkeley. Retrieved from http://escholarship.org/uc/item/3fg $3 \mathrm{k} 1 \mathrm{p} 0$

Fried, E. (2015). Problematic assumptions have slowed down depression research: why symptoms, not syndromes are the way forward. Frontiers in Psychology, 6, 309.

Fried, E., \& Nesse, R. M. (2015a). Depression is not a consistent syndrome: an investigation of unique symptom patterns in the STAR* D study. Journal of Affective Disorders, 172, 96-102.

Fried, E., \& Nesse, R. M. (2015b). Depression sum-scores don't add up: why analyzing specific depression symptoms is essential. BMC Medicine, 13(1), 72.

Hamaker, E. L., Kuiper, R. M., \& Grasman, R. P. (2015). A critique of the cross-lagged panel model. Psychological Methods, 20(1), 102-116. https://doi.org/doi.org/10.1037/a0038889

Hawkins, M. A. W., Callahan, C. M., Stump, T. E., \& Stewart, J. C. (2014). Depressive symptom clusters as predictors of incident coronary artery disease: a 15-year prospective study. Psychosomatic Medicine, 76(1), 38-43. https://doi.org/10.1097/PSY.0000000000000023

Helajärvi, H., Rosenström, T., Pahkala, K., Kähönen, M., Lehtimäki, T., Heinonen, O. J., ... Raitakari, O. T. (2014). Exploring Causality between TV Viewing and Weight Change in Young and Middle-Aged Adults. The Cardiovascular Risk in Young Finns Study. PLoS ONE, 9(7), e101860. https://doi.org/10.1371/journal.pone.0101860

Hwang, B., Moser, D. K., Pelter, M. M., Nesbitt, T. S., \& Dracup, K. (2015). Changes in Depressive Symptoms and Mortality in Patients With Heart Failure: Effects of Cognitive-Affective and Somatic Symptoms. Psychosomatic Medicine, 77(7), 798-807. https://doi.org/10.1097/PSY.0000000000000221 
Hyvärinen, A. (2010). Pairwise Measures of Causal Direction in Linear Non-Gaussian Acyclic Models. (M. Sugiyama \& Q. Yang, Eds.), JMLR: Workshop and Conference Proceedings: 2nd Asian Conference on Machine Learning (ACML2010). Tokyo, Japan. Retrieved from http://proceedings.mlr.press/v13/hyvarinen10a/hyvarinen10a.pdf

Jokela, M., García-Velázquez, R., Airaksinen, J., Gluschkoff, K., Kivimäki, M., \& Rosenström, T. (n.d.). Chronic diseases and social risk factors in relation to specific symptoms of depression: Evidence from the U. S. National Health and Nutrition Examination Surveys.

Jokela, M., Virtanen, M., Batty, G. D., \& Kivimäki, M. (2016). Inflammation and Specific Symptoms of Depression. JAMA Psychiatry, 73(1), 87. https://doi.org/10.1001/jamapsychiatry.2015.1977

Kanske, P., Heissler, J., Schönfelder, S., \& Wessa, M. (2012). Neural correlates of emotion regulation deficits in remitted depression: The influence of regulation strategy, habitual regulation use, and emotional valence. NeuroImage, 61(3), 686-693.

https://doi.org/10.1016/j.neuroimage.2012.03.089

Karp, J. F., Buysse, D. J., Houck, P. R., Cherry, C., Kupfer, D. J., \& Frank, E. (2004). Relationship of Variability in Residual Symptoms With Recurrence of Major Depressive Disorder During Maintenance Treatment. American Journal of Psychiatry, 161(10), 1877-1884. https://doi.org/10.1176/ajp.161.10.1877

Klumpp, H., Kinney, K. L., Bhaumik, R., \& Fitzgerald, J. M. (2018). Principal component analysis and brain-based predictors of emotion regulation in anxiety and depression. Psychological Medicine, 1-10. https://doi.org/10.1017/S0033291718003148

Kroenke, K., \& Spitzer, R. L. (2002). The PHQ-9: A New Depression Diagnostic and Severity Measure. Psychiatric Annals, 32(9), 509-515. https://doi.org/10.3928/0048-5713-20020901-06 
Kruis, J., \& Maris, G. (2016). Three representations of the Ising model. Scientific Reports, 6(October), 34175. https://doi.org/10.1038/srep34175

Kuiper, R. M., \& Ryan, O. (2018). Drawing Conclusions from Cross-Lagged Relationships: ReConsidering the Role of the Time-Interval. Structural Equation Modeling, 25(5), 809-823. https://doi.org/10.1080/10705511.2018.1431046

Lamers, F., Milaneschi, Y., de Jonge, P., Giltay, E. J., \& Penninx, B. W. J. H. (2018). Metabolic and inflammatory markers: associations with individual depressive symptoms. Psychological Medicine, 48(07), 1102-1110. https://doi.org/10.1017/S0033291717002483

Lawlor, D. A., Tilling, K., \& Smith, G. D. (2017). Triangulation in aetiological epidemiology. International Journal of Epidemiology, 45(6), dyw314. https://doi.org/10.1093/ije/dyw314

Lorenzo-Luaces, L. (2015). Heterogeneity in the prognosis of major depression: from the common cold to a highly debilitating and recurrent illness. Epidemiology and Psychiatric Sciences, 24(466472). https://doi.org/10.1017/S2045796015000542

Marchetti, I., Loeys, T., Alloy, L. B., \& Koster, E. H. W. (2016). Unveiling the Structure of Cognitive Vulnerability for Depression: Specificity and Overlap. PLoS ONE, 11(12), e0168612. https://doi.org/10.1371/journal.pone.0168612

Marsman, M., Borsboom, D., Kruis, J., Epskamp, S., van Bork, R., Waldorp, L. J., ... Maris, G. (2018). An Introduction to Network Psychometrics: Relating Ising Network Models to Item Response Theory Models. Multivariate Behavioral Research, 53(1), 15-35. https://doi.org/10.1080/00273171.2017.1379379

McRae, K. (2016). Cognitive emotion regulation: a review of theory and scientific findings. Current 
Opinion in Behavioral Sciences, 10, 119-124. https://doi.org/10.1016/J.COBEHA.2016.06.004

McRae, K., Ochsner, K. N., Mauss, I. B., Gabrieli, J. J. D., \& Gross, J. J. (2008). Gender Differences in Emotion Regulation: An fMRI Study of Cognitive Reappraisal. Group Processes \& Intergroup Relations, 11(2), 143-162. https://doi.org/10.1177/1368430207088035

Messerotti Benvenuti, S., Buodo, G., Mennella, R., \& Palomba, D. (2015). Somatic, but not cognitiveaffective, symptoms are associated with reduced heart rate variability in individuals with dysphoria. Frontiers in Psychology, 06, 599. https://doi.org/10.3389/fpsyg.2015.00599

Micceri, T. (1989). The unicorn, the normal curve, and other improbable creatures. Psychological Bulletin, 105(1), 156-166. https://doi.org/10.1037/0033-2909.105.1.156

Michal, M., Wiltink, J., Kirschner, Y., Wild, P. S., Münzel, T., Ojeda, F. M., ... Beutel, M. E. (2013). Differential Associations of Depressive Symptom Dimensions with Cardio-Vascular Disease in the Community: Results from the Gutenberg Health Study. PLoS ONE, 8(8), e72014. https://doi.org/10.1371/journal.pone.0072014

Munafò, M. R., \& Smith, G. D. (2018). Robust research needs many lines of evidence. Nature, 553(7689), 399-401. https://doi.org/10.1038/d41586-018-01023-3

Oud, J. H. L., \& Delsing, M. J. M. H. (2010). Continuous Time Modeling of Panel Data by means of SEM. In K. Montfort, J. Oud, \& A. Satorra (Eds.), Longitudinal Research with Latent Variables (pp. 201-244). Berlin, Heidelberg: Springer Berlin Heidelberg. https://doi.org/10.1007/978-3-64211760-2_7

Pérez, S., Marco, J. H., \& García-Alandete, J. (2016). Psychopathological Differences Between Suicide Ideators and Suicide Attempters in Patients with Mental Disorders. Clinical Psychology and 
Psychotherapy, 24, 1002-1013. https://doi.org/10.1002/cpp.2063

R Core Team. (2017). R: A Language and Environment for Statistical Computing. Vienna, Austria: R Foundation for Statistical Computing. Retrieved from https://www.r-project.org/

Roest, A. M., Carney, R. M., Freedland, K. E., Martens, E. J., Denollet, J., \& de Jonge, P. (2013).

Changes in cognitive versus somatic symptoms of depression and event-free survival following acute myocardial infarction in the Enhancing Recovery In Coronary Heart Disease (ENRICHD) study. Journal of Affective Disorders, 149(1-3), 335-341.

https://doi.org/10.1016/J.JAD.2013.02.008

Rosenström, T., \& García-Velázquez, R. (n.d.). Distribution-based causal inference: a review and practical guidance for epidemiologists. In W. Wiedermann, D. Kim, E. Sungur, \& A. Von Eye (Eds.), Direction Dependence in Statistical Models: Methods of Analysis.

Rosenström, T., Jokela, M., Puttonen, S., Hintsanen, M., Pulkki-Råback, L., Viikari, J. S., ... Keltikangas-Järvinen, L. (2012). Pairwise Measures of Causal Direction in the Epidemiology of Sleep Problems and Depression. PLoS ONE, 7(11), e50841. https://doi.org/10.1371/journal.pone.0050841

Shimizu, S., \& Bollen, K. (2014). Bayesian Estimation of Causal Direction in Acyclic Structural Equation Models with Individual-specific Confounder Variables and Non-Gaussian Distributions. Journal of Machine Learning Research, 15, 2629-2652.

Shimizu, S., Hoyer, P. O., Hyvärinen, A., \& Kerminen, A. (2006). A Linear Non-Gaussian Acyclic Model for Causal Discovery. Journal of Machine Learning Research, 7, 2003-2030. Retrieved from https://www.cs.helsinki.fi/u/ahyvarin/papers/JMLR06.pdf 
Shimizu, S., Inazumi, T., Sogawa, Y., Hyvarinen, A., Kawahara, Y., Washio, T., ... Bollen, K. (2011). DirectLiNGAM: A Direct Method for Learning a Linear Non-Gaussian Structural Equation Model. Journal of Machine Learning Research, 12, 1225-1248.

Sokero, P., Eerola, M., Rytsälä, H., Melartin, T., Leskelä, U., Lestelä-Mielonen, P., \& Isometsä, E. (2006). Decline in suicidal ideation among patients with MDD is preceded by decline in depression and hopelessness. Journal of Affective Disorders, 95, 95-102. https://doi.org/10.1016/j.jad.2006.04.028

Spirtes, P., \& Zhang, K. (2016). Causal discovery and inference: concepts and recent methodological advances. Applied Informatics, 3, 3. https://doi.org/10.1186/s40535-016-0018-x

Thamvitayakul, K., Shimizu, S., Ueno, T., Washio, T., \& Tashiro, T. (2012). Bootstrap confidence intervals in DirectLiNGAM. In IEEE 12th International Conference on Data Mining Workshops (pp. 659-668). https://doi.org/10.1109/ICDMW.2012.134

Tsai, J., Levenson, R., \& McCoy, K. (2006). Cultural and temperamental variation in emotional response. Emotion, 6(3), 484-497. https://doi.org/10.1037/1528-3542.6.3.484

van Eeden, W. A., van Hemert, A. M., Carlier, I. V. E., Penninx, B. W., \& Giltay, E. J. (n.d.). Severity, course trajectory and within-person variability of individual symptoms in patients with major depressive disorder. Acta Psychiatrica Scandinavica. https://doi.org/10.1111/acps.12987

Visted, E., Vøllestad, J., Nielsen, M. B., \& Schanche, E. (2018). Emotion Regulation in Current and Remitted Depression: A Systematic Review and Meta-Analysis. Frontiers in Psychology, 9, 756. https://doi.org/10.3389/fpsyg.2018.00756

Wiedermann, W., Kim, D., Sungur, E., \& Von Eye, A. (n.d.). Direction Dependence in Statistical 
Models: Methods of Analysis. (W. Wiedermann, D. Kim, E. Sungur, \& A. Von Eye, Eds.).

Wiedermann, W., Merkle, E. C., \& Von Eye, A. (2018). Direction of dependence in measurement error models. British Journal of Mathematical and Statistical Psychology, 71, 117-145. https://doi.org/10.1111/bmsp.12111

Wiedermann, W., \& Von Eye, A. (2016). Statistics and causality: methods for applied empirical research. (W. Wiedermann \& A. Von Eye, Eds.). New Yersey: Wiley. Retrieved from https://www.wiley.com/enus/Statistics+and+Causality\%3A+Methods+for+Applied+Empirical+Research-p-9781118947043

Wiltink, J., Michal, M., Jünger, C., Münzel, T., Wild, P. S., Lackner, K. J., ... Beutel, M. E. (2018). Associations between degree and sub-dimensions of depression and metabolic syndrome (MetS) in the community: results from the Gutenberg Health Study (GHS). BMC Psychiatry, 18(1), 114. https://doi.org/10.1186/s12888-018-1691-1

Wiltink, J., Michal, M., Wild, P. S., Schneider, A., König, J., Blettner, M., ... Beutel, M. E. (2014). Associations between Depression and Diabetes in the Community: Do Symptom Dimensions Matter? Results from the Gutenberg Health Study. PLoS ONE, 9(8), e105499. https://doi.org/10.1371/journal.pone.0105499

Zhang, K., Gong, M., Ramsey, J., Batmanghelich, K., Spirtes, P., \& Glymour, C. (2017). Causal Discovery with Linear Non-Gaussian Models under Measurement Error: Structural Identifiability Results. Retrieved from http://auai.org/uai2018/proceedings/papers/372.pdf 
Table 1. Sampling-weighted item frequencies and sample descriptive information. NHANES 2005$2015(\mathrm{n}=34963)$.

\begin{tabular}{|c|c|c|c|c|c|}
\hline \multicolumn{2}{|l|}{ Symptom } & Not at all & Several days & $\begin{array}{l}\text { More than } \\
\text { half of the } \\
\text { days }\end{array}$ & $\begin{array}{c}\text { Nearly every } \\
\text { day }\end{array}$ \\
\hline \multicolumn{2}{|c|}{ Anhedonia/loss of interest } & .77 & .16 & .04 & .03 \\
\hline \multicolumn{2}{|l|}{ Depressed mood } & .78 & .16 & .03 & .03 \\
\hline \multicolumn{2}{|c|}{ Feelings of worthlessness/guilt } & .83 & .12 & .03 & .02 \\
\hline \multicolumn{2}{|c|}{ Thoughts of death/self-harm } & .97 & .02 & .01 & $<.01$ \\
\hline \multicolumn{3}{|l|}{ Variable } & \multicolumn{3}{|l|}{ Sample } \\
\hline Gender & Fem & & 52 & & \\
\hline Age & Mea1 & & $46.24(17.41)$ & & \\
\hline \multirow[t]{5}{*}{ Race-ethnicity } & \multicolumn{2}{|c|}{ Mexican American, \% } & 9 & & \\
\hline & \multicolumn{2}{|c|}{ Other Hispanic, $\%$} & \multicolumn{3}{|l|}{5} \\
\hline & \multicolumn{2}{|c|}{ Non-Hispanic white, $\%$} & \multicolumn{3}{|l|}{67} \\
\hline & \multicolumn{2}{|c|}{ Non-Hispanic black, \% } & \multicolumn{3}{|l|}{12} \\
\hline & \multicolumn{2}{|c|}{ Other race, $\%$} & \multicolumn{3}{|l|}{7} \\
\hline $\begin{array}{l}\text { Ratio of family income } \\
\text { to poverty }\end{array}$ & \multicolumn{2}{|c|}{ Below poverty threshold, $\%$} & \multicolumn{3}{|l|}{15} \\
\hline
\end{tabular}


Table 2. Confirmatory test for vanishing tetrads and bootstrapped p-values.

\begin{tabular}{|l|l|l|}
\hline Cohort & T statistic(df), p-value & Bootstrapped p-value \\
\hline 2005 & $14.111(2), \mathrm{p}<.001$ & $<.0001$ \\
\hline 2007 & $14.099(2), \mathrm{p}<.001$ & .0010 \\
\hline 2009 & $10.511(2), \mathrm{p}=0.003$ & .0025 \\
\hline 2011 & $14.188(2), \mathrm{p}<.001$ & $<.0001$ \\
\hline 2013 & $33.144(2), \mathrm{p}<.001$ & $<.0001$ \\
\hline 2015 & $36.913(2), \mathrm{p}<.001$ & $<.0001$ \\
\hline
\end{tabular}


Table 3. Total significant effects of symptoms on columns over symptoms on rows.

\begin{tabular}{|c|c|c|c|c|c|}
\hline Cohort & Symptoms & $\begin{array}{l}\text { Anhedonia/ } \\
\text { loss interest }\end{array}$ & $\begin{array}{l}\text { Depr. } \\
\text { mood }\end{array}$ & Worthlessness/guilt & $\begin{array}{l}\text { Thoughts of } \\
\text { death and self- } \\
\text { harm }^{\text {a }}\end{array}$ \\
\hline 2005 & \multirow{6}{*}{$\begin{array}{l}\text { Anhedonia/loss of } \\
\text { interest }\end{array}$} & 1 & 0 & .40 & .79 \\
\hline 2007 & & 1 & .40 & .42 & .57 \\
\hline 2009 & & 1 & .48 & .48 & .56 \\
\hline 2011 & & 1 & .50 & .43 & .67 \\
\hline 2013 & & 1 & .48 & .46 & .53 \\
\hline 2015 & & 1 & .48 & .42 & .64 \\
\hline 2005 & \multirow{6}{*}{$\begin{array}{l}\text { Depressed } \\
\text { mood }\end{array}$} & & 1 & .59 & .91 \\
\hline 2007 & & & 1 & .57 & .88 \\
\hline 2009 & & & 1 & .61 & .80 \\
\hline 2011 & & & 1 & .56 & .83 \\
\hline 2013 & & & 1 & .60 & .83 \\
\hline 2015 & & & 1 & .61 & .88 \\
\hline 2005 & \multirow[t]{6}{*}{ Worthlessness/guilt } & & & 1 & .82 \\
\hline 2007 & & & & 1 & .76 \\
\hline 2009 & & & & 1 & .67 \\
\hline 2011 & & & & 2 & .77 \\
\hline 2013 & & & & 1 & .88 \\
\hline 2015 & & & & 1 & .92 \\
\hline
\end{tabular}

Footnote: ${ }^{a}$ The variable "Thoughts of death and self-harm" was strictly exogenous, meaning only dominant. 
Directed acyclic graph displaying the associations estimated by DirectLiNGAM in six cross-sectional samples drawn from NHANES 2005-2015 $(n=34$ 963).

Feelings of worthlessness or guilt

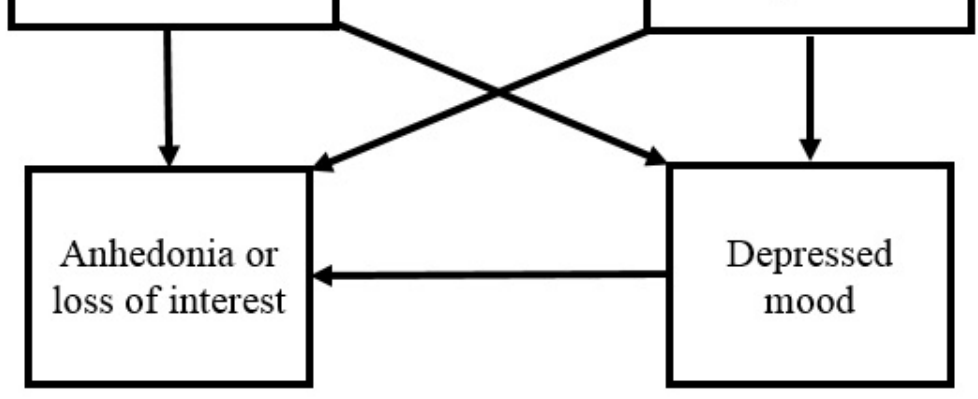

$122 \times 78 \mathrm{~mm}(144 \times 144$ DPI $)$ 